International Journal of Pure and Applied Mathematics

Volume 90 No. 1 2014, 25-34

ISSN: 1311-8080 (printed version); ISSN: 1314-3395 (on-line version)

url: http://www.ijpam.eu

doi: http://dx.doi.org/10.12732/ijpam.v90i1.4

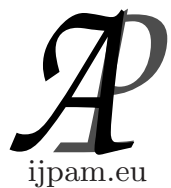

\title{
TWO CASES OF RECONSTRUCTION OF SEPARABLE GRAPHS
}

\author{
Martin Dowd \\ 1613 Wintergreen $\mathrm{Pl}$. \\ Costa Mesa, CA 92626, USA
}

\begin{abstract}
The author has shown that a separable graph with more than one block which is not an edge is reconstructible. Here reconstructibility is proved for a single such block, in two particular cases.
\end{abstract}

AMS Subject Classification: $05 \mathrm{C} 60$

Key Words: reconstructibility, separability

\section{Introduction}

Say that a vertex $v$ is indicated in a tree if the tree is equipped with a $0-1$ labeling of the vertices which is 1 only at $v$. Define a twig of a graph $G$ to be an edge incident to a vertex of degree 1 . If $G$ is not a tree, the trunk of $G$ is defined to be the graph obtained by successively removing twigs until none remain. Define a limb of $G$ to be a maximal subtree, with the vertex contained in the trunk indicated. Note that $G$ is the trunk, with the limbs attached at distinct vertices.

In [4] it is shown that trees are reconstructible. In [1] it is shown that the limbs of a separable graph are reconstructible. In [3] it is shown that if the trunk of $G$ has more than one block then $G$ is reconstructible.

Received: September 9, 2013

(c) 2014 Academic Publications, Ltd. url: www.acadpubl.eu 
From hereon, assume that $G$ has a single block $B$ which is not an edge, and at least one limb. By the size of a limb is meant its number of edges. Note that $B$ can be determined, by considering any $G_{v}$ where $v$ is a degree 1 vertex.

Theorem 1. If there is an $s>1$ such that there are limbs with $s$ vertices but none with $s-1$ vertices then $G$ is reconstructible.

Proof. This was observed in [1]. For a proof, let $v$ be any vertex such that $G_{v}$ has a limb of size $s-1$. $G$ may be reconstructed by replacing the limb of size $s-1$ by the missing limb of size $s$.

A limb of size 1 will be called a 1-limb. From hereon it will be assumed that there is at least one 1-limb. Let $\mathcal{T}_{d s}$ be the multiset of limbs of size $s$ attached to vertices if degree $d$.

Theorem 2. Except in the case of a single limb of size 1, the family of $\mathcal{T}_{d s}$ is reconstructible.

Proof. If there is no 1-limb the result follows by theorem 1 . Let $v$ be a degree 1 vertex of a 1 -limb; then each $\mathcal{T}_{d s}$ for $s>1$ may be determined from $G_{v}$. When there is more that one 1-limb it is readily seen that the family of $\mathcal{T}_{d 1}$ may be determined from this family in $G_{v}$ as $v$ ranges over the degree 1 vertices of 1-limbs. In there is a single 1-limb and some other limb, let $v$ be a degree 1 vertex of a limb of least size greater than 1 ; then in $G_{v}$ there are one or two 1-limbs, and the degree of the vertex where the 1-limb occurs in $G$ may be determined in either case.

\section{The Case of a Cycle}

In this section let $B$ be a cycle with $b$ vertices. Let $k$ be the number of limbs of size greater than 1 .

Lemma 3. Suppose $k \geq 3$. Then $G$ is reconstructible.

Proof. Let $v$ be the degree 1 vertex of a 1-limb. In $G_{v}$ let $P_{1}$ be the path between roots of limbs of size greater than 1 , which includes all such roots, and let $P_{2}$ be the other path between the endpoints of $P_{1}$. Choose an orientation of $P_{1}$, and let $T_{1}, \ldots, T_{k}$ be the limbs of size greater than 1 in order of their roots along $P_{1}$. Let $p_{t}$ be the number of vertices in $P_{t}$ for $t=1,2$. If $p_{1} \neq p_{2}$ or $k \geq 4$ or $T_{2}$ has more than 2 vertices, a vertex $w$ can be found in a $T_{i}$ with $1<i<k$, so that $P_{1}$ can be found in $G_{w}$. If there is no ambiguity in the orientation of $P_{1}$ 
in $G_{w}, G$ can be reconstructed by replacing $T_{i}-w$ in $G_{w}$ with $T_{i}$. Otherwise, the $T_{j}$ must be symmetric, with $k$ odd and $i=(k+1) / 2$. Again the graph may be reconstructed by replacing $T_{i}-w$ in $G_{w}$ with $T_{i}$.

Say that $w$ is good if it is a degree 1 vertex of some $T_{t}$, and it can be determined in $G_{w}$ which limb equals $T_{t}-w$. If $w$ is good then $G$ may be reconstructed by replacing $T_{j}-w$ in $G_{w}$ by $T_{t}$.

Suppose $p_{1}=p_{2}, k=3$, and $T_{2}$ has 2 vertices. Let $t_{t}$ be the size of $T_{t}$ for $t=1,3$. Let $j$ be such that $t_{j} \leq t_{4-j}$. If $t_{j} \geq 3$ then a good vertex may be found in $T_{j}$. If $t_{4-j} \geq 4$ then a good vertex may be found in $T_{4-j}$. If $t_{j}=2$ and $t_{4-j}=3$. a good vertex may be found in $T_{j}$.

In the remaining case, $t_{j}=2$ and $t_{4-j}=2$. If either $T_{1}$ or $T_{3}$ is different from $T_{2}$, a good vertex may be found in $T_{t}$ for $t=1$ or $t=3$, by ensuring that $T_{4-j}$ differs from $T_{2}$. Let $p_{12}$ be the distance from the root of $T_{1}$ to the root of $T_{2}$, and similarly for $p_{23}$. If $p_{12} \neq p_{23}$ then a good vertex may be found in $T_{1}$ (or $T_{3}$ ).

If $p_{12}=p_{23}$, choose $w$ in $T_{2}$. If only one path from the root of $T_{1}$ to the root of $T_{3}$ has an edge attached to its midpoint, replace the edge with $T_{2}$. Otherwise let $w$ be the degree 1 vertex of the edge attached to the midpoint of $T_{2} ; w$ can be found, and an edge attached to the correct vertex in $G_{w}$.

Lemma 4. Suppose $k=2$. Then $G$ is reconstructible.

Proof. Let $T_{1}, T_{2}$ be the limbs and $t_{1}, t_{2}$ their sizes. By theorem 1, it mat be assumed that either $t_{1}=2$ and $t_{2}=3$, or $t_{1}=t_{2}=2$. Let $p_{1} \leq p_{2}$ be the lengths of the paths between the roots of $T_{1}$ and $T_{2}$; these may be determined from $G_{v}$ where $v$ is the degree 1 vertex of any 1 -limb.

If $p_{1}=p_{2}$ let $v$ be a degree 1 vertex of $T_{1}$. In $G_{v}$ the 1 -limb which needs to be replaced by $T_{1}$ may be determined, so $G$ may be reconstructed by performing the replacement. By considering degree 1 vertices of 1 -limbs it may be determined whether one of the paths has no 1-limbs attached. If so let $v$ be the degree 1 vertex of $T_{1}$. In $G_{v}$, let $r$ be the root of $T_{2}$ in $G_{v}$ and let $s$ be the root of a 1-limb, such that one of the paths between $r$ and $s$ contains all roots of 1-limbs. $G$ may be reconstructed by replacing the 1 -limb with root $s$ by $T_{1}$.

Suppose $p_{1}<p_{2}$ and both paths have 1-limbs attached. By considering the $G_{v}$ with $v$ a degree 1 vertex of a 1-limb, $P_{1}$ and $P_{2}$ may be determined, where these are the paths between the roots of $T_{1}, T_{2}$, with $P_{i}$ of length $p_{i}$, with all limbs attached. If both $P_{1}$ and $P_{2}$ contain a single 1-limb, let $v$ be a degree 1 vertex of $T_{1}$; in $G_{v}$, replace the "middle" 1 -limb by $T_{1}$.

Otherwise, let $P$ be one of $P_{1}, P_{2}$ with more than 1 1-limb. Let $t_{i}$ be the 1-limb nearest $T_{i}$, and let $d_{i}$ be the distance between the roots of $t_{i}$ and $T_{i}$. If 
$d_{1}=d_{2}$ let $v$ be the degree 1 vertex of either $t_{1}$ or $t_{2} ; G$ may be reconstructed from $G_{v}$ by adding a 1-limb at the appropriate position in $P$. Otherwise, renumbering if needed, suppose $d_{1}<d_{2}$. Let $t_{3}$ be the next 1 -limb after $t_{1}$ starting at $T_{1}$. if $d_{3} \neq d_{2}$ then $v$ may be chosen as the degree vertex of $t_{1}$, and $t_{1}$ added to $G_{v}$. If $d_{3}=d_{2}$ then $v$ may be chosen as the degree 1 vertex of $t_{2}$ (even if $t_{2}=t_{3}$ ), and $t_{2}$ added to $G_{v}$.

Lemma 5. Suppose $k=1$. Then $G$ is reconstructible.

Proof. Let $T$ be the limb of size greater than 1 ; by theorem $1 T$ may be assumed to have size 2 . Let $v$ be a vertex of degree 1 in $T$. If there is only 1 1-limb $t$, the two distances from $t$ to $T$ may be determined by considering $G_{v}$. Let $w$ be the degree 1 vertex of $t$; then $t$ may be added to $G_{w}$. If there is more than 1 -limb, the two closest to $T$ may be found, say $t_{1}$ and $t_{2}$, with distances $d_{1} \leq d_{2}$. The rest of the argument is a variation of the argument given for $P$ in the proof of lemma 4.

Lemma 6. Suppose $k=0$. Then $G$ is reconstructible.

Proof. Let $v$ range over the roots of 1-limbs. If for any $G_{v}$ the path is of length $b-2$ or $b, G$ may be reconstructed from such a $G_{v}$. Otherwise, $G$ consists of the circular concatenation of paths of length 3 , where the degree 1 vertices have no 1-limb and the degree 2 vertices have a 1-limb.

Theorem 7. If $B$ is a cycle then $G$ is reconstructible. If there are limbs of size greater than 1 then $G$ is reconstructible from $\left\{G_{v}: v\right.$ is a vertex of degree $1\}$.

Proof. The first claim follows by lemmas 3, 4, 5, and 6. The second claim follows by examining the proofs.

\section{The Case of 3 Paths}

It is well-known (proposition 3.1.1 of [2]) that any 2-connected graph can be built up from a cycle by successively attaching paths. Thus, a natural case to consider for $B$ is that of a cycle, with one path added. Such a graph may be characterized as a pair of vertices, with 3 paths between them; or as a graph with two vertices of degree 3 and all other vertices of degree 2 .

Suppose $B$ is such a graph. Let $a \leq b \leq c$ be the path lengths. Call a limb an end limb if it is attached to a degree 3 vertex, else an internal limb. 
By theorem 2, except in the case of a single limb of size 1, the end limbs and internal limbs are determined.

Lemma 8. If there is a single limb, of size 1, then $G$ is reconstructible.

Proof. Let $v$ range over the degree 3 vertices. Let $w$ be the degree 1 vertex of the limb. If the limb is an end limb, attach a 1-limb to a degree 3 vertex in $G_{w}$. Otherwise, choose one of the $G_{v}$, and let $\alpha, \beta, \gamma$ be the path lengths. There is exactly one of the values $a, b, c$ which is not among $\alpha+1, \beta+1, \gamma+1$; let $x$ denote it. $G$ may be reconstructed from $G_{v}$ by adding a vertex, adding an edge to the neighbor of the end of a path of length $x$, and adding edges to the ends of the other paths.

Lemma 9. If there are any end limbs then $G$ is reconstructible.

Proof. Let $e$ be the sum of the sizes of the end limbs. Suppose $e \geq 2$. Let $v$ be a degree 1 vertex of a smaller of the two end limbs (or the only end limb). The end at which the missing end limb needs to be attached is readily determined.

Suppose $e=1$; by lemma 8 it may be assumed that there are internal limbs. Letting $s$ denote the sum of the sizes on a path, let $P$ be such that $s$ takes its least nonzero value. Let $w$ be a degree 1 vertex of a limb of $P$, closest to the end with the limb attached; $G$ is readily reconstructed from $G_{w}$.

For the rest of the section, suppose all limbs are internal. Let $s$ be the sum of the sizes of the limbs on a path. Writing a letter for $s \geq 2$, there are 14 possibilities for the size triples in nondecreasing order: 001, 00r, 011, 01r, 0rr, 0rs, 111, 11r, 1rr, 1rs, rrr, rrs, rss, rst

Each size triple gives rise to a list of size triples, of the $G_{v}$ where $v$ ranges over degree 1 vertices. By direct computation, these lists are distinct, and the sizes determined, except in the case 002 and 011 . Which of these occurs is known since the limbs are. The case 001 has already been proved.

Lemma 10. If the size triple contains two 0's then $G$ is reconstructible.

Proof. Let $P$ denote the path containing limbs. If there are limbs of size greater than 1 , let $v$ be a degree 1 vertex in such a limb, and let $w$ be the degree 1 vertex of a 1-limb. It is readily verified that $G$ can be reconstructed from $G_{v}$ by comparing $P$ in $G_{v}$ and $G_{w}$.

Suppose all limbs are 1-limbs. Since 001 is already proved, $P$ may be located in $G_{v}$ where $v$ is an end vertex. If there is no missing 1-limb in $G_{v}$ connect a 
new vertex to all end vertices of the paths in $G_{v}$. Otherwise, connect the new vertex to the neighbor of the end vertex of $P$ instead.

Lemma 11. If the size triple contains one 0 then $G$ is reconstructible.

Proof. By letting $v$ range over degree 1 vertices, the length $l_{0}$ of the path with no limbs may be determined.

Suppose the size triple is $01 r$, and all limbs are 1-limbs. Let $v$ range over the end vertices. If there is a $v$ such that no limbs are missing in $G_{v}, G$ may be reconstructed from $G_{v}$ by adding a vertex and edges to the ends of all 3 paths. If there is a $v$ such that two limbs are missing in $G_{v}, G$ may be reconstructed from $G_{v}$ by adding a vertex, an edge to a path of length $l_{0}-1$, and edges to the neighbors of the ends of the other 2 paths. If for both $v$ one limb is missing in $G_{v}$, choose the one with the most 1-limbs on one of the paths; add an edge to the end of this path, and the neighbor of the end of the other path.

Suppose the size triple is 012 , and there is a limb of size 2. Proceed as in the previous case, where the limb of size 2 is considered missing if it is either completely missing or has become a 1-limb.

Suppose the size triple is $01 r$ where $r$ is greater than 2, and there is a limb of size greater than 1 . Let $w$ be the degree 1 vertex of the 1-limb $t$ on the path containing just this limb. Let $v_{1}$ be a degree 1 vertex of a limb $T_{1}$ of size greater than 1 , of distance $d$ from the closest end vertex. In $G_{w}$, the path $P$ of size $r$ may be determined. In $G_{v_{1}}$, if this may be laid over the path of size $a-1$ in only one way, $G$ has been reconstructed. Otherwise, there is a limb $T_{2}$ at distance $d$ from the other end, and the remaining limbs are symmetric. If $T_{1}=T_{2}$ or $t$ is at the midpoint of its path then $P$ may be overlaid either way. Otherwise the choice may be made by considering $G_{v_{1}}$ and $G_{v_{2}}$.

Suppose the size triple is $0 \mathrm{rr}$ or $0 \mathrm{rs}$, and all limbs are 1-limbs. Proceed as in the case of $01 r$. If for both $v$ one limb is missing in $G_{v}$, choose the one with the smallest number of limbs on a path. Add an edge to the neighbor of the end vertex of the path of size $r-1$, and to the end of the longer path.

Suppose the size triple is $0 \mathrm{rr}$ or $0 \mathrm{rs}$, and there are limbs of size greater than 1. Let $C$ be the cycle of the two paths with limbs. $C$ may be determined in any $G_{v}$ where $v$ is a degree 1 vertex. The argument of theorem 7 may be adapted. Examination of the proof shows that only the case of a single limb of size 2 and a single 1-limb requires further argument. This case has already been handled.

Lemma 12. If the size triple of $G$ is 111 then $G$ is reconstructible. 
Proof. Let $v$ be an end vertex. In $G_{v}$ connect an added vertex to the ends of paths of size 0 , and the neighbor of the end in paths of size 1 .

Lemma 13. If the size triple of $G$ is $11 r$ then $G$ is reconstructible.

Proof. Suppose $r \geq 3$. Let $w$ be a degree 1 vertex of a 1-limb on a path of size 1. Let $v$ be a degree 1 vertex of a limb on the path $P$ of size $r$. $P$ may be determined from $G_{w}$. The other two paths $C$ may be determined from $G_{v}$. If $C$ or $P$ is symmetric $G$ is readily reconstructed from $G_{v}$. In the remaining case the orientation of $P$ overlaying $P$ in $G_{v}$ is determined.

Suppose $r=2$. If there is a limb of size 2 and the length of its path is greater than 2, let $v$ be an end vertex where there is a limb of size 2 in $G_{v} ; G$ is readily reconstructed from $G_{v}$. If there is a limb of size 2 and the length of its path is 2 , let $v$ be a degree 1 vertex of the limb of size 2 in $G_{v} ; G$ is readily reconstructed from $G_{v}$. Suppose all limbs are 1-limbs; let $v$ range over the end vertices. If there is a $v$ such that in $G_{v}$ there is a path of size $2, G$ is readily reconstructed from $G_{v}$. Otherwise let $v$ be such that the number of 1-limbs adjacent to an end vertex is as large as possible; $G$ is readily reconstructed from $G_{v}$.

Lemma 14. If the size triple of $G$ is $1 \mathrm{rr}$ then $G$ is reconstructible.

Proof. Suppose $r \geq 3$. Let $w$ be the degree 1 vertex of the 1 -limb $t$ on the path $P$ of size 1 . Let $v$ be a degree 1 vertex of a limb on a path of size $r$. The paths $C$ of size $r$, with their ends marked, may be determined from $G_{w}$. The path $P_{1}$ of size 1 may be determined from $G_{v}$. If the limb is at the middle of $P_{1}, G$ may be reconstructed from $G_{w}$.

Otherwise, there are 2 or 4 possible limbs in $G_{w}$ which can cover the missing limb in $G_{v}$, according to whether it is at the middle of its path or not, in accordance with the possibilities for covering $C$ in $G_{v}$. Some of these possibilities may be eliminated sine only some of the possible coverings are allowed. The remaining ones which occur in $G_{v}$ may also be eliminated, leaving a single possibility.

Suppose $r=2$. Let $w$ be as above, and let $l$ be the length of $P_{1}$. If $l=2$ then $G$ is readily reconstructed from $G_{v}$. If $l \geq 4$ there is a vertex $x$ where $G_{x}$ is a cycle with a limb of size at least $3 ; G$ is readily reconstructed from $G_{x}$.

If $l=3$ there is an end vertex $v$ where $G_{v}$ has a path of length 2 with a 1-limb. Now, it is readily verified that if $v_{1}$ and $v_{2}$ are the end vertices of $C$. If $C_{v_{1}}$ and $C_{v_{2}}$ are isomorphic then $C$ is symmetric. In this case a 1-limb may be added at either internal vertex of the path of size 0 in $G_{w}$. Otherwise, $C_{v}$ in 
$G_{v}$ can be matched with the correct $C_{v_{t}}$, and $G$ is readily reconstructed from $G_{v}$.

Lemma 15. If the size triple of $G$ is $1 r s$ then $G$ is reconstructible.

Proof. Let $w$ be the degree 1 vertex of the 1-limb $t$ on the path $P$ of size 1. Let $v$ be a degree 1 vertex of a limb on the path of length $s$. The paths of size greater than 1 , with their ends marked, may be determined from $G_{w}$. The path $P_{1}$ of size 1 may be determined from $G_{v}$. If the limb is at the middle of $P_{1}, G$ may be reconstructed from $G_{w}$.

If $r \geq 3$ let $v_{1}$ be a degree 1 vertex of a limb $T_{1}$ of the path of length $r$. If $G_{w}$ may be laid over $G_{v_{1}}$ then $G$ is reconstructed. Otherwise, $T_{1}$ has an alternative $T_{t}$; which should be chosen can be determined, since $t$ is not in the middle of $T_{1}$.

If $r=2$ let $x$ be the end vertex nearest $t$. By considering $G_{y}$ for $y$ a degree 1 vertex of a limb on the path of length $r$, the distance of these limb(s) from $x$ may be determined.

Lemma 16. If the size triple is $r r r$, then $G$ is reconstructible.

Proof. Let $v$ range over degree 1 vertices. In each $G_{v}$ there is a cycle $C$ with two paths of size $r$. Up to isomorphism, there are 1, 2, or 3 distinct cycles. Orient each of these, so that there is a top and bottom branch, and a left and right end vertex. Let $P^{r}$ denote the reverse of the path $P$.

If there is only one cycle $C$, a second copy may be matched to the first as either $C_{b}=C_{t}, C_{b}=C_{t}^{r}, C_{b}=C_{b}^{r}$, or $C_{b}=C_{b}^{r}$. If $C_{b}=C_{t}$ then all 3 paths are equal. If $C_{b}=C_{t}^{r}$ then all 3 paths are equal and symmetric. If $C_{b}=C_{b}^{r}$ then all 3 paths are equal. If $C_{b}=C_{b}^{r}$ then all 3 paths are equal and symmetric.

If there are two cycles $C_{1}$ and $C_{2}$ the cases are as follows. In each case the pattern of the paths $C_{1 t}, C_{1 b}$, and the third path are given. $C_{1 b}=C_{2 t}: A B B$; $C_{1 b}=C_{2 t}^{r}: A B B ; C_{1 b}=C_{2 b}: A B B ; C_{1 b}=C_{2 b}^{r}: A B B ; C_{1 b}=C_{1 t}:$ impossible; $C_{1 b}=C_{1 t}^{r}: A A^{r} A ; C_{1 b}=C_{1 b}: A B A ; C_{1 b}=C_{1 b}^{r}: A a^{r} A$. Note that additional equalities might hold, such as $B=A^{r}$, as long as there remain two distinct cycles.

If there are three cycles $C_{1}, C_{2}, C_{3}$, by relabeling it may be assumed that $C_{3 t}=C_{1 t}=A, C_{2 t}=C_{1 b}=B$, and $C_{3 b}=C_{2 b}=C$. The possibilities for $C_{1 b}$ are $C_{2 t}, C_{2 t}^{r}, C_{2 b}, C_{2 b}^{r}, C_{1 t}, C_{1 t}^{r}, C_{1 b}$, and $C_{1 b}^{r}$. These merely impose additional restrictions on $A, B, C$, some of which are impossible (such as equality of any two).

Lemma 17. If the size triple is rrs, then $G$ is reconstructible. 
Proof. Let $v$ range over degree 1 vertices of paths of size $r$. In each $G_{v}$ the cycle $C$ of the paths of size $r$ in $G$ may be identified. The argument of theorem 7 may be adapted. The limbs are not all 1-limbs, and the exceptional case of a single 1-limb and a single limb of size 2 does not occur.

Lemma 18. If the size triple is rss, then $G$ is reconstructible.

Proof. Let $v$ be a degree 1 vertex in a limb attached to the path $P$ of length $r$. In $G_{v}$ the cycle $C$ of the paths of length $s$, with their ends marked, may be found. The number $n$ of limbs on $P$ may also be determined.

Suppose $n \geq 3$. Letting $T_{1}$ and $T_{2}$ denote the limbs closest to the end vertices, with the root of $T_{i}$ at distance $d_{i}$ from the closest end, by considering $G_{v}$ as $v$ ranges over degree 1 vertices in limbs on $P, T_{1}, d_{1}, T_{2}$, and $d_{2}$ may be determined. Now let $w$ be a degree 1 vertex in one of the $T_{i}$, and replace the limb $T_{i}-w$ (which may be empty) by $T_{i}$ (noting that $T_{3-1}$ is known).

Suppose $n=2$. Unless both limbs are 1-limbs, the argument for $n \geq 3$ applies. Suppose both limbs are 1-limbs. If $s \geq 4$ than $P$ with all limbs attached may be determined. This may be laid over $P$ in $G_{v}$ where $v$ is a degree 1 vertex of a limb on $P$. The same argument applies if $s=3$ and either path of length 3 has a limb of size greater than 1 .

Thus, suppose $r=2, s=3$, and all limbs are 1-limbs. Let $v$ be the root of the middle 1-limb on a path of size 3 ; it is readily verified that $G$ is reconstructible from $G_{v}$.

Suppose $n=1$. The case $r=1$ has already been handled. Otherwise, let $v$ be a degree 1 vertex in the limb $T$ on $P$, and replace $T-v$ by $T$.

Lemma 19. If the size triple is $r$ st, then $G$ is reconstructible.

Proof. Let $P$ be the path of size $r$. Letting $v$ be a degree 1 vertex of a limb attached to $P$, the other two paths, with the end vertices marked, can be determined from $G_{v}$. Letting $w$ be a degree 1 vertex of a limb attached to the path of length $t, P$ with its end vertices marked, may be determined. If either $P$ or the other two paths are symmetric, $P$ can be overlaid in $G_{v}$ arbitrarily. Otherwise, the choice of orientation of $P$ may be determined by an argument as in the proof of lemma 11.

Theorem 20. If $B$ is a 3 path graph then $G$ is reconstructible.

Proof. This follows by lemmas 8 to 19 . 


\section{Concluding Remarks}

It is recognizable whether $G$ is a cycle, i.e., if all vertices are of degree 2 . $G$ is clearly reconstructible. It is recognizable whether $G$ is a 3 path graph, i.e., if there are 2 vertices of degree 3 and other vertices have degree 2. $G$ is reconstructible in this case also. Let $v$ be a degree 3 vertex; $G$ is reconstructible from $G_{v}$, unless there is a path of length 1 . In this case the sum of the lengths of the other two paths $P_{1}$ and $P_{2}$ is known from $G_{v}$, and the length of one of them may be determined from $G_{w}$ where $w$ is on the other.

Theorem 21. Suppose $G$ is a 2-connected graph obtained by adding $k$ paths. Then $k=e-v$ where $e$ is the number of edges and $v$ the number of vertices.

Proof. The proof is an easy induction on $k$.

Since the dimension of the cycle space over the two element field is $e-v+1$, this quantity equals $k+1$.

Adding another path to a 3 path graph, there are 3 possibilities, which may be characterized in terms of the degree sequence of the vertices of degree greater than 2. The possibilities are:

\section{4,$4 ; \quad 4,3,3 ; \quad 3,3,3,3$}

\section{References}

[1] J.A. Bondy, On Ulam's conjecture for separable graphs, Pacific J. Math., 31 (1969), 281-288, doi: 10.2140\%2Fpjm.1969.31.281.

[2] R. Diestel, Graph Theory, Springer, Germany, 2010.

[3] M. Dowd, Strong reconstructibility of the block-cutpoint tree, Int. J. Pure Appl. Math., To Appear.

[4] P. Kelly, A congruence theorem for trees, Pacific J. Math., 7 (1957), 61-68, doi: $10.2140 \% 2 F p j m .1957 .7 .961$. 